OPEN ACCESS

Edited by: Rich Boden, University of Plymouth,

United Kingdom

Reviewed by:

Bärbel Ulrike Fösel,

Helmholtz Zentrum München,

Germany

Cesar Hugo

Hernández-Rodríguez,

National Polytechnic Institute, Mexico

${ }^{*}$ Correspondence:

Eiko E. Kuramae

E.Kuramae@nioo.knaw.nl

Specialty section:

This article was submitted to Microbial Physiology and Metabolism,

a section of the journal

Frontiers in Microbiology

Received: 27 February 2020

Accepted: 14 May 2020

Published: 18 June 2020

Citation:

Costa OYA, Oguejiofor $C$, Zühlke D, Barreto CC, Wünsche C,

Riedel K and Kuramae EE (2020) Impact of Different Trace Elements on

the Growth and Proteome of Two

Strains of Granulicella, Class

"Acidobacteriia".

Front. Microbiol. 11:1227.

doi: 10.3389/fmicb.2020.01227

\section{Impact of Different Trace Elements on the Growth and Proteome of Two Strains of Granulicella, Class "Acidobacteriia"}

\author{
Ohana Y. A. Costa ${ }^{1,2}$, Chidinma Oguejiofor ${ }^{3}$, Daniela Zühlke ${ }^{4}$, Cristine C. Barreto5, \\ Christine Wünsche ${ }^{4}$, Katharina Riedel ${ }^{4}$ and Eiko E. Kuramae ${ }^{1,6 *}$
}

${ }^{1}$ Department of Microbial Ecology, Netherlands Institute of Ecology (NIOO-KNAW), Wageningen, Netherlands, ${ }^{2}$ Institute of Biology Leiden, Leiden University, Leiden, Netherlands, ${ }^{3}$ Department of Soil Science and Meteorology, Michael Okpara University of Agriculture, Umudike, Nigeria, ${ }^{4}$ Institute of Microbiology, University of Greifswald, Greifswald, Germany, ${ }^{5}$ Genomic Sciences and Biotechnology Program, Catholic University of Brasilia, Distrito Federal, Brazil, ${ }^{6}$ Ecology and Biodiversity, Institute of Environmental Biology, Utrecht University, Utrecht, Netherlands

Acidobacteria represents one of the most dominant bacterial groups across diverse ecosystems. However, insight into their ecology and physiology has been hampered by difficulties in cultivating members of this phylum. Previous cultivation efforts have suggested an important role of trace elements for the proliferation of Acidobacteria, however, the impact of these metals on their growth and metabolism is not known. In order to gain insight into this relationship, we evaluated the effect of trace element solution SL10 on the growth of two strains (5B5 and WH15) of Acidobacteria belonging to the genus Granulicella and studied the proteomic responses to manganese (Mn). Granulicella species had highest growth with the addition of $\mathrm{Mn}$, as well as higher tolerance to this metal compared to seven other metal salts. Variations in tolerance to metal salt concentrations suggests that Granulicella sp. strains possess different mechanisms to deal with metal ion homeostasis and stress. Furthermore, Granulicella sp. 5B5 might be more adapted to survive in an environment with higher concentration of several metal ions when compared to Granulicella sp. WH15. The proteomic profiles of both strains indicated that Mn was more important in enhancing enzymatic activity than to protein expression regulation. In the genomic analyses, we did not find the most common transcriptional regulation of $\mathrm{Mn}$ homeostasis, but we found candidate transporters that could be potentially involved in Mn homeostasis for Granulicella species. The presence of such transporters might be involved in tolerance to higher $\mathrm{Mn}$ concentrations, improving the adaptability of bacteria to metal enriched environments, such as the decaying wood-rich Mn environment from which these two Granulicella strains were isolated.

Keywords: Acidobacteria, Granulicella, genome, proteome, manganese, metabolism 


\section{INTRODUCTION}

Despite being widespread and dominant in soil ecosystems (Kuramae et al., 2012; Navarrete et al., 2013b; Pereira de Castro et al., 2016), the phylum Acidobacteria has a low number of cultivated representatives, due to difficulties in isolation and propagation under laboratory conditions (Dedysh and Yilmaz, 2018). Most Acidobacteria isolates are slow growers and can take weeks to months to develop colonies (Eichorst et al., 2011; de Castro et al., 2013). Recently, changes in traditional culture methods and application of unconventional culture media composition have increased the number of new Acidobacteria isolates considerably. Currently, 62 species with validated names have been described (NCBI Resource Coordinators, 2016), while in 2011 only 14 species had been isolated and characterized (de Castro, 2011). Modifications in culture media and cultivation conditions, such as low concentration of nutrients (Janssen et al., 2002; Stevenson et al., 2004), higher $\mathrm{CO}_{2}$ concentrations (Stevenson et al., 2004), unusual or complex polysaccharides as carbon sources (Pankratov et al., 2008; Eichorst et al., 2011), longer incubation periods (de Castro et al., 2013), addition of humic acids and quorum-sensing molecules (Stevenson et al., 2004), employment of soil solution equivalents and inhibitors for unwanted microorganisms (de Castro et al., 2013; Foesel et al., 2013), are strategies that have been applied for the enrichment and isolation of new Acidobacteria species.

Once the isolates are obtained, better cell proliferation can be achieved with richer culture media, containing higher concentrations of nutrients (de Castro et al., 2013). For instance, trace elements can be used to improve microbial growth and gain of biomass under laboratory conditions, even though the specific requirements among strains and species are variable (Banerjee et al., 2009; Merchant and Helmann, 2012). Metal ions such as Fe, $\mathrm{Mn}, \mathrm{Zn}$, and $\mathrm{Cu}$ are fundamental for microbial metabolism, being required at low concentrations (Abbas and Edwards, 1990). They play an important role in biological processes, acting as co-factors of enzymes (Wintsche et al., 2016), activating metalloregulators and trace element dependent proteins (Hantke, 2001; Zhang et al., 2009), forming functional complexes with secondary metabolites (Morgenstern et al., 2015; Locatelli et al., 2016) and promoting the detoxification of reactive oxygen species (ROS) (Kehres and Maguire, 2003; Locatelli et al., 2016).

Although some culture media used for Acidobacteria growth and isolation are supplemented with trace elements (de Castro et al., 2013; Navarrete et al., 2013a), the impact of these metals on their growth and metabolism is not yet known. Though metal ions are essential for many biological processes, they can be toxic at high concentrations (Puri et al., 2010). Metal ions cannot be synthesized or degraded, therefore cellular homeostasis of metals relies mostly on transport, which involves several mechanisms that sense, uptake, immobilize or pump metals out of the cell (Chandrangsu et al., 2017). In the present study, we evaluated the effect of trace elements and particularly Mn on the growth of Granulicella spp. WH15 and 5B5, Acidobacteria (class “Acidobacteriia”), derived from decaying wood (Valášková et al., 2009). We used the optimized culture medium PSYL5 (Campanharo et al., 2016) to boost the growth of the two strains, evaluated the impact of Mn through proteome studies and performed genomic analyses on both strains.

\section{MATERIALS AND METHODS}

\section{Acidobacteria Strains}

Two strains of Acidobacteria, 5B5 and WH15 belonging to Granulicella genus of class "Acidobacteriia" were used in this study. Both strains belong to the culture collection of Netherlands Institute of Ecology (NIOO-KNAW), Department of Microbial Ecology. They were isolated from wood in advanced decay stage, in association with the white-rot fungus Hypholoma fasciculare, in Netherlands (Valášková et al., 2009). The genome of Granulicella sp. WH15 is deposited at NCBI with accession number CP042596 while the genome of Granulicella sp. 5B5 was sequenced in this study.

\section{Trace Elements Solution (SL10) and Individual Trace Elements Effect on Bacterial Growth}

The effect of trace element solution SL10 (Widdel et al., 1983) on the growth of both bacterial strains was evaluated for two different concentrations ( 1 and $10 \mathrm{ml}$ ) of the solution per $\mathrm{L}$ of PSYL5 culture medium. PSYL5 medium was composed of (g/L): $1.8 \mathrm{KH}_{2} \mathrm{PO}_{4}, 0.2 \mathrm{MgSO}_{4} .7 \mathrm{H}_{2} \mathrm{O}, 30$ sucrose and 1.0 yeast extract; $\mathrm{pH}$ was adjusted to 5.0 (Campanharo et al., 2016). Culture medium without the amendment of SL10 solution was used as a control. Seven-day-old cell suspensions of both strains were inoculated in $70 \mathrm{ml}$ of culture medium to an $\mathrm{OD}_{600 \mathrm{~nm}} 0.01$. The cultures were incubated under aeration for 7 days at $30^{\circ} \mathrm{C}$ and a constant rotation rate of $50 \mathrm{rpm}$. Every $24 \mathrm{~h}$ the optical density of the cultures was measured with an Eppendorf photometer at a wavelength of $600 \mathrm{~nm}$ (Eppendorf, Hamburg, Germany). For the evaluation of the different trace elements on the growth of both strains, individual trace element stock solutions and growth curves were executed for each metal separately, using the same growth conditions described above. The composition of the trace element solution SL10 and the final concentration of each trace element in the culture medium is shown in Supplementary Table S1. The metal salt that produced a significantly higher growth in comparison with the control was selected for further experiments. All experiments were executed in triplicates.

Statistical analysis was performed using SigmaPlot v14. Normality of the data was checked using Shapiro-Wilk test. Two-way repeated measures ANOVA was used to test the effect of SL10 solution and individual trace elements on the growth rate of Granulicella spp. WH15 and 5B5.

\section{Genome of Granulicella sp. 5B5}

The Granulicella sp. 5B5 strain obtained from the collection of Netherlands Institute of Ecology (NIOO-KNAW) was grown on 1/10 TSB agar medium (Valášková et al., 2009) at $\mathrm{pH}$ 5.0 for 3 days at $30^{\circ} \mathrm{C}$. The bacterial cells were harvested and the genomic DNA was extracted using MasterPure ${ }^{\mathrm{TM}}$ DNA Purification Kit (Epicentre, Madison, WI, United States) 
according to manufacturer's instructions. A total of $10 \mu \mathrm{g}$ of DNA was sent to the Genomics Resource Center (Baltimore, $\mathrm{MD}$, United States) for a single long insert library (15-20 kb), that was constructed and sequenced in one SMRTcell using the PacBio RS II (Pacific Biosciences, Inc.) sequencing platform. De novo assembly was performed with the help of SMRT Analysis software v2.2.0 (Pacific Biosciences) featuring HGAP 2 (Chin et al., 2013), and subsequent correction with Pilon 1.16 (Walker et al., 2014) to reveal a circular replicon: a 3,928,701 bp chromosome $(\mathrm{G}+\mathrm{C}$ content $61,1 \% ; 58 \times$ coverage). Automatic gene prediction and annotation was performed by using Prokka (Seemann, 2014) and RAST genome annotation server ${ }^{1}$ (Aziz et al., 2008). Predicted genes were mapped to Cluster of Ortholog Groups (COG) and KEGG IDs using the COG database (2014 release) (Galperin et al., 2015) and KEGG database (release 2013) (Kanehisa, 2000), using eggNOG mapper. The CAZyme contents of Granulicella sp. 5B5 genome were determined by identifying predicted genes containing CAZyme domains using the dbCAN2 meta server ${ }^{2}$ (Zhang et al., 2018), according to the CAZy (Carbohydrate-Active Enzyme) database classification (Lombard et al., 2014). Only CAZyme domains predicted by at least two of the three algorithms (DIAMOND, HMMER, and Hotpep) employed by dbCAN2 were kept. The phylogenetic relationship between Granulicella sp. 5B5, Granulicella sp. WH15, and other Granulicella species was analyzed based on $16 \mathrm{~S}$ rRNA gene sequences. The dendrogram was constructed by using Maximum-Likelihood [Tamura-Nei model (Tamura and Nei, 1993), 1000 bootstraps] analysis, with software MEGA X (Kumar et al., 2018). Pairwise genome alignment was performed using the lastz v. 1.04 program (Harris, 2007). The results were visualized using AliTV v. 1.0.6. (Ankenbrand et al., 2017). Circular genome map was drawn using CGView software (Stothard and Wishart, 2004). Average Nucleotide Identity (ANI) between Granulicella spp. 5B5 and WH15 was calculated using the webtool ANI calculator, available at https://www.ezbiocloud. net/tools/ani (Yoon et al., 2017).

\section{Metal Tolerance Assays and Metal Resistance Gene Annotation}

The metal tolerance of Granulicella spp. 5B5 and WH15 to varied metal salt concentrations was tested in solid culture medium (Abou-Shanab et al., 2007; Afzal et al., 2017) PSYL5 pH 5. Five concentrations $(0.5,1,2,5$, and $10 \mathrm{mM})$ of nine metal salts were tested: $\mathrm{ZnCl}_{2}, \mathrm{NiCl}_{2}, \mathrm{MnCl}_{2}, \mathrm{CoCl}_{2}, \mathrm{CuCl}_{2}, \mathrm{NaMoO}_{4}, \mathrm{AlCl}_{2}$, $\mathrm{CdCl}_{2}$, and $\mathrm{C}_{8} \mathrm{H}_{4} \mathrm{~K}_{2} \mathrm{O}_{12} \mathrm{Sb}_{2}$ (Abou-Shanab et al., 2007; Terry et al., 2015). As a control, an Escherichia coli DH5 $\alpha$ strain, with known low metal tolerance was used. Six isolated colonies of each strain previously grown on PSYL5 solid medium without metal were streaked on the culture media with each different metal concentration. Growth was interpreted as positive when colony formation was similar to growth on control culture medium without the addition of metals. After 7 days of growth at $30^{\circ} \mathrm{C}$, colonies were streaked on a new plate with the same metal concentration, in order to confirm growth. If colonies did not

${ }^{1}$ http://rast.nmpdr.org/

${ }^{2}$ http://bcb.unl.edu/dbCAN2/ develop within 7 days, plates were incubated for extra 7 days. Colonies were restreaked three times for confirmation. When the strains were resistant to the highest concentration of metal used (10 mM), we performed additional tests using higher metal concentrations $(15,20,25,30$, and $40 \mathrm{mM})$.

In order to identify predicted genes that could be involved in metal ion homeostasis, we searched the genomes of both strains against the experimentally confirmed and predicted BacMet databases using BacMet Scan (Pal et al., 2014) with less strict parameters ( $40 \%$ similarity), due to the high quantity of hypothetical proteins in the genomes of both bacteria.

\section{Acquisition of Cytosolic Proteome With and Without Manganese Treatment by Mass Spectrometry and Data Analysis}

For the proteome analysis, we analyzed the effects of the metal salt which significantly improved the growth yield of both strains in comparison to the control without metal salt. Therefore, Mn was selected for further experiments. The growth curves of Granulicella spp. WH15 and 5B5 with added manganese $\left(\mathrm{MnCl}_{2}\right)$ and controls without trace elements were repeated, using the same parameters as described in section "Trace Elements Solution (SL10) and Individual Trace Elements Effect on Bacterial Growth." Cells were collected at day 4 of the growth curve, when the differences in the $\mathrm{OD}_{600 \mathrm{~nm}}$ between manganese treatment and control treatment started to be statistically significant. A total of $3 \mathrm{ml}$ of bacterial cell culture per replicate $(n=3$ for each treatment) were harvested by centrifugation at $10,015 \times g$ at $4^{\circ} \mathrm{C}$ for $10 \mathrm{~min}$. Pellets were washed twice with $1 \mathrm{~mL}$ of $\mathrm{TE}$ buffer and finally resuspended in $1 \mathrm{ml} \mathrm{TE} \mathrm{buffer.} \mathrm{A} \mathrm{volume}$ of $500 \mu \mathrm{L}$ of cell suspension was transferred into $2 \mathrm{~mL}$ screw cap tubes filled with $500 \mu \mathrm{L}$ glass beads $(0.1 \mathrm{~mm}$ in diameter; Sarstedt, Germany) and mechanically disrupted using Fastprep (MP Biomedicals) for $3 \times 30 \mathrm{~s}$ at $6.5 \mathrm{~m} / \mathrm{s}$; with on ice incubation for $5 \mathrm{~min}$ between cycles. To remove cell debris and glass beads, samples were centrifuged for $10 \mathrm{~min}$ at $4^{\circ} \mathrm{C}$ at $21,885 \times g$, followed by a second centrifugation $\left(30 \mathrm{~min}\right.$ at $4^{\circ} \mathrm{C}$ at $\left.21,885 \times g\right)$ to remove insoluble and aggregated proteins. The protein extracts were kept at $-20^{\circ} \mathrm{C}$. Protein concentration was determined using RotiNanoquant (Carl Roth, Germany). Proteins were separated by SDS-PAGE. Protein lanes were cut into 10 equidistant pieces and in-gel digested using trypsin as described earlier (Grube et al., 2014). Tryptic peptides were separated on an EASY-nLC II coupled to an LTQ Orbitrap Velos using a non-linear binary 76 min gradient from 5 to $75 \%$ buffer B $(0.1 \%$ acetic acid in acetonitrile) at a flow rate of $300 \mathrm{~nL} / \mathrm{min}$ and infused into an LTQ Orbitrap Velos (Thermo Fisher Scientific, United States) mass spectrometer. Survey scans were recorded in the Orbitrap at a resolution of 60,000 in the $\mathrm{m} / \mathrm{z}$ range of $300-1,700$. The 20 most-intense peaks were selected for CID fragmentation in the LTQ. Dynamic exclusion of precursor ions was set to $30 \mathrm{~s}$; single-charged ions and ions with unknown charge were excluded from fragmentation; internal calibration was applied (lock mass 445.120025).

For protein identification resulting MS/MS spectra were searched against a database containing protein sequences of 
Granulicella sp. strain 5B5 or Granulicella sp. strain WH15 and common laboratory contaminants $(9,236$ entries or 7,782 entries, respectively) using Sorcerer-Sequest v.27, rev. 11 (Thermo Fisher Scientific) and Scaffold v4.7 (Proteome Software, United States) as described earlier (Stopnisek et al., 2016). Relative quantification of proteins is based on normalized spectrum abundance factors (NSAF; Zhang et al., 2010).

Statistical analysis was done using MeV (Saeed et al., 2003); $t$-test was applied for proteins that were identified in at least two replicates of the respective condition. Hierarchical clustering and $t$-test of $\mathrm{z}$-transformed normalized data were performed with the following parameter unequal group variances were assumed (Welch approximation), $p$-values based on all permutation with $p=0.01$, significance determined by adjusted Bonferroni correction. Only significantly changed proteins showing at least 1.5-fold changes between conditions were considered for further analysis. Furthermore, so-called on/off proteins, that were only identified in one condition were analyzed. Functional classification of Granulicella sp. strain 5B5 and WH15 proteins was carried out using eggNOG mapper ${ }^{3}$ (Huerta-Cepas et al., 2017), COG (Galperin et al., 2015), and KEGG databases (Kanehisa, 2000). In order to identify proteins that could be involved in metal ion homeostasis, we searched proteins with significantly changed amounts against the experimentally confirmed and predicted BacMet databases using BacMet Scan (Pal et al., 2014) with less strict parameters ( $40 \%$ similarity), due to the high quantity of hypothetical proteins in the genomes of both bacteria. Voronoi treemaps for visualization of proteome data were generated with Paver software (Decodon GmbH, Germany).

\section{RESULTS}

\section{Trace Mineral Solution (SL10) Effects on Growth}

The addition of trace element solution SL10 in liquid culture medium produced a significant effect $(p<0.001)$ on the growth of both strains of Granulicella sp. Both concentrations $(1 \times$ and $10 \times$ ) of trace element solution (SL10) significantly increased Granulicella sp. 5B5 growth, with the highest growth recorded for $1 \times$ and $10 \times$ concentrations of SL10 (Figure 1A). In addition, the cultures showed a longer lag phase for $10 \times$ concentration of SL10 (Figure 1A).

Granulicella sp. WH15 had a significantly higher growth rate with the addition of $1 \times$ SL10 compared to control and $10 \times$ SL10 (Figure 1B), except at day one of incubation. Differently from Granulicella sp. 5B5, 10× SL10 did not enhance the growth of Granulicella sp. WH15, having instead the opposite effect (Figure 1B).

\section{Effect of Individual Trace Element on Growth}

Of all the trace elements, manganese $(\mathrm{Mn})$ and copper $(\mathrm{Cu})$ significantly increased the growth of Granulicella sp. 5B5

${ }^{3}$ http://eggnogdb.embl.de/\#/app/emapper compared to control starting from day three of the incubation period until the end $(p<0.001)$ (Figure 1C). Iron (Fe) significantly increased the growth of Granulicella sp. 5B5 only at day 6, when compared with the control. Boron (B), zinc ( $\mathrm{Zn})$, cobalt (Co), nickel (Ni), and molybdate (Mo) did not have any significant effect on the growth of the strain throughout the duration of the experiment (Figures 1C,D).

Throughout the incubation period, Mn was the only trace element that significantly $(p<0.001)$ increased the growth of Granulicella sp. WH15 in comparison with the control with no metal (Figure 1E). Fe, $\mathrm{Zn}, \mathrm{Co}, \mathrm{Cu}, \mathrm{Ni}, \mathrm{Mo}$, and Bo did not have any significant effect on the growth of Granulicella sp. WH15 as compared to the control (Figures 1E,F).

\section{Granulicella sp. 5B5 Genome Annotation and CAZymes}

The assembled genome of Granulicella sp. $5 \mathrm{~B} 5$ is 3,928,701 bp, with $61.1 \%$ GC content, 3,306 proteins and only one rRNA operon. Functional annotation using COG and RAST analysis resulted in the classification of 2,615 predicted genes into 20 COG functional groups and the annotation of 1,260 predicted genes to RAST subsystems. The properties of the genomes of Granulicella sp. 5B5 and also Granulicella sp. WH15 (sequenced previously, Costa et al., 2020b) are listed in Table 1. A circular genome map of Granulicella sp. 5B5 is depicted in Supplementary Figure S1, together with that of strain WH15. The distribution of predicted genes into COGs/RAST functional categories for Granulicella sp. 5B5 genome is depicted in Figure 2. ANI (Figueras et al., 2014) between Granulicella spp. WH15 and 5B5 was $72.75 \%$, showing that the strains do not belong to the same species. Comparisons among $16 \mathrm{~S}$ sequences demonstrated that Granulicella sp. 5B5 is more similar to Granulicella cerasi and Granulicella paludicola, while Granulicella sp. WH15 is closer to Granulicella tundricola and Granulicella rosea (Supplementary Figure S2A). Genome alignment between the strains showed several genomic rearrangements and few regions of genome similarity (Supplementary Figure S2B).

RAST analysis showed that only $37 \%$ of the annotated genes $(1,260 / 3,374)$ could be assigned to subsystems. Among the subsystem categories present in the genome, "carbohydrates" and "dormancy and sporulation" had the highest and lowest feature counts, respectively (Supplementary Figure 2B).

Analysis with ANTISMASH v4.2.0 revealed the presence of five biosynthetic gene clusters (Table 3 ). The identified clusters showed potential for the production of terpenes, beta-lactone, type III polyketide synthases (T3PKS) and bacteriocin. Only cluster 1 showed similarity to a known cluster (Malleobactin NRPS, 11\% similarity). Annotation with dbCAN (Table 2) revealed the presence of 92 carbohydrate-associated enzymes, distributed in four classes: seven carbohydrate esterases (CE), 63 glycosyl hydrolases (GH), 20 glycosyl transferases (GT), and two polysaccharide lyases (PL), but no carbohydrate binding modules (CBM) or auxiliary activities (AA) were observed. Further evaluation of the CAZymes demonstrated the potential for the degradation of a wide range of carbohydrates, as 


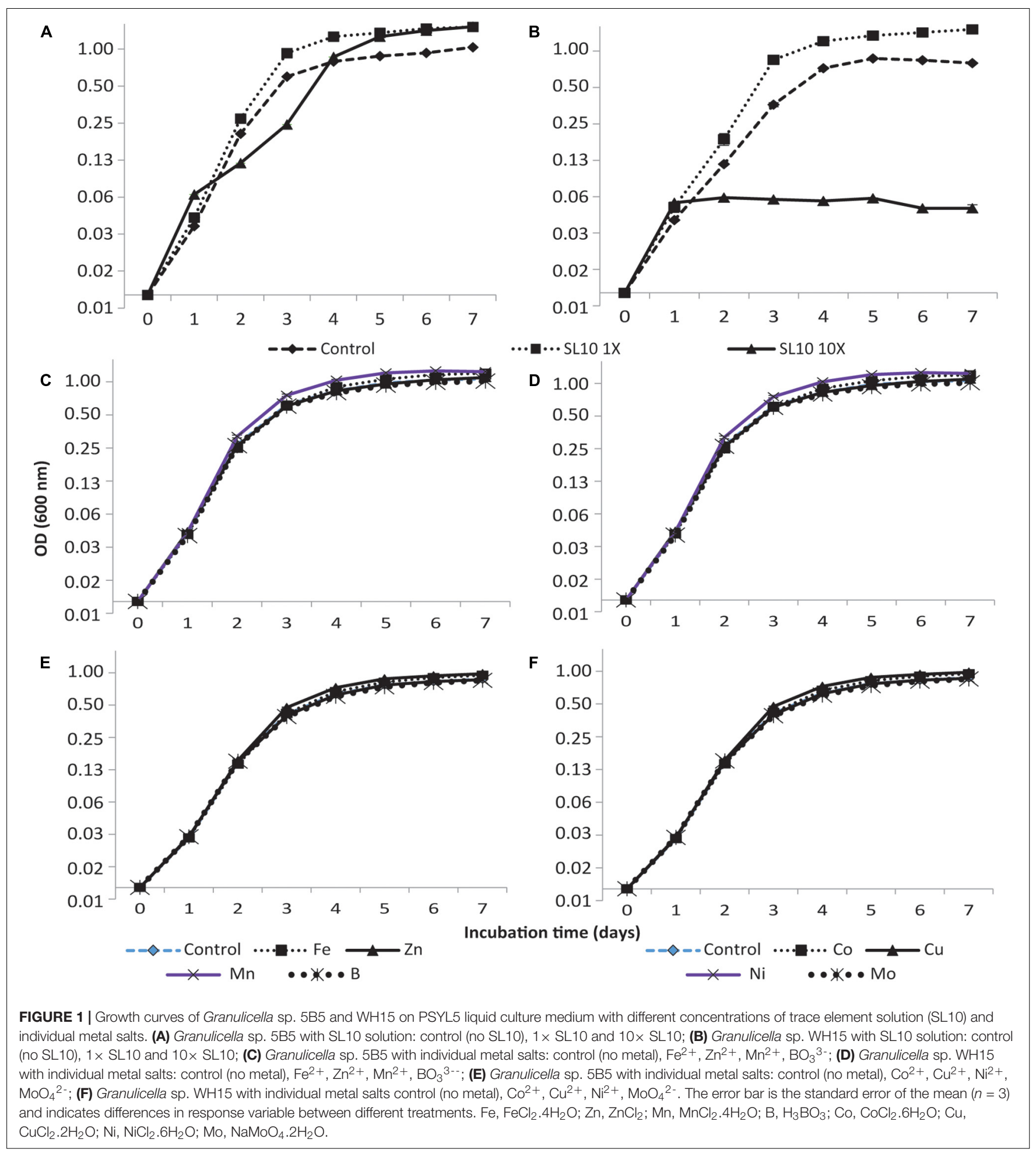

the genome of Granulicella sp. 5B5 possessed CDSs for 48 CAZyme families, including as $\alpha$ - and $\beta$-glucosidases (EC 3.2.1.20, EC 3.2.1.21) (GH1, GH13, GH3, GH31), $\alpha$ - and $\beta$-galactosidases (EC 3.2.1.22, EC 3.2.1.23) (GH2, GH27, GH35, GH57), $\alpha$ - and $\beta$-mannosidases (EC 3.2.1.25, EC 3.2.1.25) (GH1, GH38, GH125), $\alpha$-L-rhamnosidases (EC 3.2.1.40) (GH28,
GH106), $\alpha$-L-fucosidases (EC 3.2.1.51) (GH29), $\beta$-xylosidases (EC 3.2.1.37) (GH39, GH43, GH54), $\alpha$-L-arabinofuranosidases (EC 3.2.1.55) (GH43, GH54) and $\alpha$-amylases (EC 3.2.1.1) (GH13, GH77). The cellulose synthase genes observed in other Granulicella genomes (Rawat et al., 2013a,b) were not observed in the genome of Granulicella sp. 5B5. 
TABLE 1 | Genomic features of Granulicella sp. 5B5 and WH15.

\begin{tabular}{lcc}
\hline Genome & $\begin{array}{c}\text { Granulicella } \\
\text { sp. 5B5 }\end{array}$ & $\begin{array}{c}\text { Granulicella } \\
\text { sp. WH15 }\end{array}$ \\
\hline Size (bp) & $3,928,701$ & $4,673,153$ \\
G + C content (\%) & 61.1 & 60.7 \\
Number of predicted coding sequences & 3,306 & 3,939 \\
Number of features in Subsystems & 1,260 & 1,496 \\
Number of predicted RNA genes & 51 & 51 \\
Number of contigs & 1 & 1 \\
\hline
\end{tabular}

\section{Metal Resistance Assays and Metal Resistance Gene Annotation}

Metal resistance tests on agar medium demonstrated that both Granulicella strains were able to grow on $\mathrm{NiCl}_{2}(\max 2 \mathrm{mM})$ and $\mathrm{NaMoO}_{4}(\max 1 \mathrm{mM})$. Granulicella sp. 5B5 was able to grow on $0.5 \mathrm{mM} \mathrm{ZnCl}_{2}$, Granulicella sp. WH15 grew on $0.5 \mathrm{AlCl}_{2}$, and both strains could grow at concentrations of $\mathrm{MnCl}_{2}$ up to $40 \mathrm{mM}$ (Table 3).

Granulicella sp. WH15 genome search against BacMet experimentally confirmed and predicted resistance genes databases revealed 28 ORFs and 78 ORFS, respectively, with hits similar ( $>45 \%$ identity) to genes involved in resistance toward a wide range of metals such as $\mathrm{As}, \mathrm{Cd}, \mathrm{Zn}, \mathrm{Co}, \mathrm{Cu}, \mathrm{Fe}, \mathrm{Mn}, \mathrm{Mo}$, $\mathrm{Ni}$, and $\mathrm{Zn}$, multidrug and metal efflux transporters and DNA binding response regulators (Supplementary Table S2).

In addition, Granulicella sp. WH15 possessed two non-identical copies of Mn transporter $m n t H$ (TC 2.A.55.3.1) (GWH15_03215 and GWH15_08180), and two ORFs (GWH15_19170 and GWH15_03225), with 60.2 and 44\% identity with the $\mathrm{Mn}$ transcriptional regulator $m n t R$, respectively.

We obtained a similar profile for Granulicella sp. 5B5 genome, with 65 ORFs that had hits higher than 45\% identity against the experimentally confirmed database and 23 ORFs that had hits higher than $45 \%$ identity against the predicted database (Supplementary Table S3). For both searches, predicted genes involved in resistance to several metal ions, as well as multidrug and metal efflux transporters and transcription regulators were observed (Supplementary Table S3). The genome of Granulicella sp. 5B5 also contains two non-identical copies of the mntH transporter (TC 2.A.55.3.1) (G5B5_04510 and G5B5_07650), and three ORFs (G5B5_10215, G5B5_13625, and G5B5_13770) related to $\mathrm{Mn}$ transcriptional regulator mntR, as well as three ORFs similar to $\mathrm{Mn} A B C$ transporters $m n t A / y \operatorname{tgA}$ (TC 3.A.3.3.3) (G5B5_11150 and G5B5_14665) and Mn efflux pump mntP (TC 2.A.107.1.1) (G5B5_01505) (Supplementary Table S3).

\section{Impact of Manganese on Proteome of Granulicella Strains}

Since Mn had a significant effect on the growth of both Granulicella strains, we further investigated the effects of $\mathrm{Mn}$ on cellular metabolism by proteomics analysis. At day 4, the differences in growth between control and Mn treatment started to be statistically significant for both strains
(Supplementary Figure S3), and therefore samples were collected at this particular time point for proteome analysis.

Proteome data for Granulicella sp. 5B5 showed that 1,028 proteins were detected in both treatments in at least two out of three replicates each. Overall, 216 proteins showed significantly different abundances, with 14 so-called on/off proteins, which were present in only one condition (Supplementary Figure S4A). The proteome patterns of Granulicella sp. 5B5 under control and Mn treatments are depicted in Supplementary Figure S5A. A total of 46 proteins were upregulated 1.5-fold and 11 proteins were "on," while 43 proteins were downregulated 1.5-fold and 3 proteins were "off" in the Mn treatment. Among these differentially expressed proteins, 90 could be assigned to COG categories and 67 could be annotated to KEGG orthologs (Figure 3 and Supplementary Table S4).

The qualitative analysis of the proteomic data for Granulicella sp. WH15 demonstrated that, overall, 909 proteins were identified in both conditions in two out of three replicates each. In total, 171 proteins showed significant differences between $\mathrm{Mn}$ and control conditions ( $t$-test, $p=0.01$ ) (Supplementary Figure S4B). The proteome patterns of Granulicella sp. WH15 under control and Mn treatments are depicted in Supplementary Figure S5B.

Comparisons between treatments showed that 16 proteins were upregulated at least 1.5 -fold, while 93 proteins were downregulated at least 1.5 -fold. In addition, 19 proteins were "off" in the Mn treatment and present only in control conditions. Among the significantly different proteins, 112 were assigned to COG categories and 89 were annotated to KEGG orthologs (Figure 4 and Supplementary Table S4).

Comparatively, proteome analysis revealed different responses to $\mathrm{Mn}$ for the two strains. Granulicella sp. 5B5 had more upregulated proteins (57), while Granulicella sp. WH15 had more downregulated proteins (112). Further comparisons demonstrated that no upregulated or downregulated proteins were shared between strains. In Granulicella sp. 5B5, a higher number of upregulated proteins that can use $\mathrm{Mn}^{2+}$ as cofactor was detected. For both strains, proteins annotated as Mn transporters were not detected, probably because the method used for protein extraction was not specific for membrane proteins. A model summarizing the cellular processes involved in Mn adaptation for both Granulicella sp. WH15 and Granulicella sp. 5B5 is depicted in Figure 5.

\section{Upregulated Proteins in Response to Manganese Granulicella sp. 5B5}

Cluster of Ortholog Groups analysis showed that proteins were mainly distributed among the categories E-amino acid transport and metabolism (7), L-replication, recombination and repair (5), G-carbohydrate transport and metabolism (4), and F-nucleotide transport and metabolism (4) (Figure 3A). A total of 36 proteins were assigned to KEGG orthologs, that were mapped to 47 metabolic pathways, and some orthologs were mapped to more than one pathway. The majority of the proteins were mapped to "general" metabolic pathways (35), biosynthesis 
A

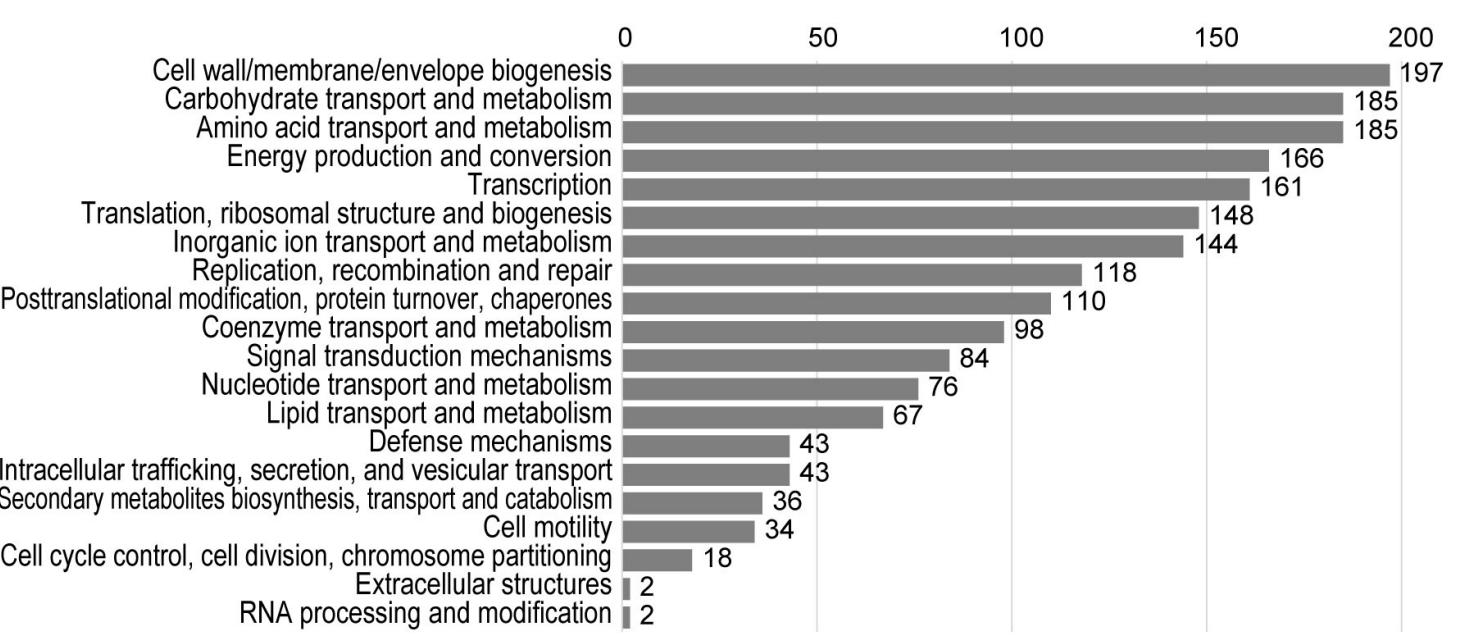

B

Subsystems coverage

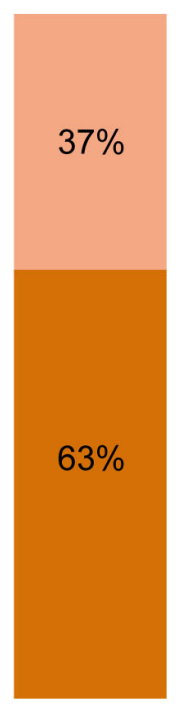

Subsystems category distribution

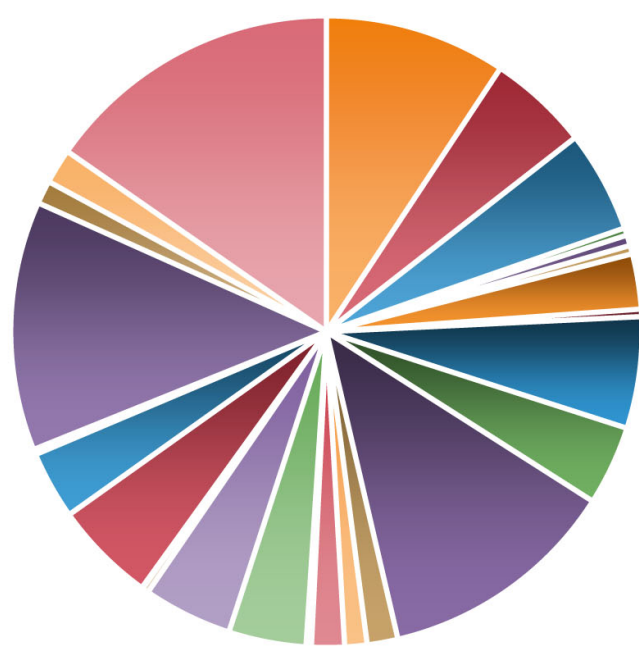

Subsystems feature counts

\author{
- Cofactors, Vitamins, Prosthetic Groups, Pigments (171) \\ - Cell Wall and Capsule (95) \\ - Virulence, Disease and Defense (96) \\ - Potassium metabolism (7) \\ - Miscellaneous (10) \\ - Phages, Prophages, Transposable elements, Plasmids (8) \\ - Membrane Transport (52) \\ - Iron acquisition and metabolism (7) \\ - RNA Metabolism (105) \\ - Nucleosides and Nucleotides (75) \\ - Protein Metabolism (227) \\ - Cell Division and Cell Cycle (29) \\ - Motility and Chemotaxis (21) \\ - Regulation and Cell signaling (31) \\ - Secondary Metabolism (5) \\ -DNA Metabolism (73) \\ -Fatty Acids, Lipids, and Isoprenoids (84) \\ - Nitrogen Metabolism (6) \\ - Dormancy and Sporulation (1) \\ - Respiration (96) \\ -Stress Response (64) \\ - Metabolism of Aromatic Compounds (5) \\ - Amino Acids and Derivatives (235) \\ - Sulfur Metabolism (22) \\ - Phosphorus Metabolism (33) \\ -Carbohydrates (282)
}

FIGURE 2 | Statistics of COG and RAST subsystems annotations of Granulicella 5B5. (A) COG categories distribution, showing number of predicted genes annotated in each category. (B) Subsystem category distribution. The light orange bar represents the percentage of proteins that could be annotated by RAST Server and the dark orange bar represents the proteins that were not annotated. The pie chart represents the percentage of proteins annotated to each subsystem category. Following the pie chart clockwise, subsystem categories are listed in the legend from top to bottom.

of antibiotics (16), biosynthesis of secondary metabolites (13), and microbial metabolism in diverse environments (10) (Figure 3B), but no metabolic pathway was specifically upregulated. Looking deeper into the upregulated proteins, we identified several enzymes that require $\mathrm{Mn}^{2+}$ or $\mathrm{Mg}^{2+}$ as cofactor, such as nucleoside diphosphate kinase Ndk (EC 2.7.4.6), octaprenyl diphosphate synthase IspB (EC 2.5.1.90), UDP-N-acetylmuramate-L-alanyl-gamma-D-glutamyl-meso-

2,6-diaminoheptandioate ligase $\mathrm{Mpl}$ (EC 6.3.2.45), adenine deaminase Ade (EC 3.5.4.2), phosphate-specific transport system accessory protein PhoU, oxalate decarboxylase OxdD (EC 4.1.1.2), phosphoenolpyruvate carboxykinase pckG (EC 4.1.1.32) and $3^{\prime}-5^{\prime}$ exoribonuclease YhaM (EC:3.1.-.-) (Supplementary Table S4).
Search against BacMet Databases showed 25 proteins with hits ( $>45 \%$ identity) against the experimentally confirmed database and 17 proteins with hits ( $>45 \%$ identity) against the predicted metal resistance genes database (Supplementary Table S5). The genes were mostly associated resistance/homeostasis of several metal ions, such as $\mathrm{Fe}, \mathrm{Cu}, \mathrm{As}, \mathrm{Ni}, \mathrm{Co}$, and $\mathrm{Zn}$. Interestingly, four ORFS were similar to metal ion transporters that could be involved in Mn homeostasis: ORF_05650 (hypothetical protein) had $43.5 \%$ identity with copper-translocating P-type ATPase CueA (TC 3.A.3.5.39); ORF_03225 (hypothetical protein) had $31 \%$ identity with copper-translocating P-type ATPase CopA (TC 3.A.3.5.1); ORF_06495 (TcrA) had 40\% identity with copper-translocating P-type ATPase CopA (TC 3.A.3.5.1) and ORF_14875 (NatA_2) had 31\% identity with metal 
TABLE 2 | Number of predicted genes from different CAZyme families observed in the genomes of Granulicella sp. 5B5 and WH15.

\begin{tabular}{lcc}
\hline CAZyme family & $\begin{array}{c}\text { Granulicella } \\
\text { sp. 5B5 }\end{array}$ & $\begin{array}{c}\text { Granulicella } \\
\text { sp. WH15 }\end{array}$ \\
\hline Auxiliary activity (AA) & 0 & 13 \\
Carbohydrate binding module (CBM) & 0 & 22 \\
Carbohydrate esterase (CE) & 7 & 41 \\
Cohesin & 0 & 1 \\
Glycoside hydrolase (GH) & 63 & 86 \\
Glycosyl transferase (GT) & 20 & 52 \\
Polysaccharide lyase (PL) & 2 & 2 \\
Total & $\mathbf{9 2}$ & $\mathbf{2 1 7}$ \\
\hline
\end{tabular}

TABLE 3 | Growth of Granulicella sp. WH15 and 5B5 in solid culture medium with different metal concentrations.

\begin{tabular}{|c|c|c|c|c|c|c|c|}
\hline \multirow[t]{2}{*}{ Metal source } & \multirow[t]{2}{*}{ Strain } & \multicolumn{6}{|c|}{ Concentration in $\mathrm{mM}$} \\
\hline & & 0.5 & 1 & 2 & 5 & 10 & $15-40$ \\
\hline & WH15 & & & & & & \\
\hline $\mathrm{ZnCl}_{2}$ & & - & - & - & - & - & - \\
\hline $\mathrm{NiCl}_{2}$ & & + & + & + & - & - & - \\
\hline $\mathrm{MnCl}_{2}$ & & + & + & + & + & + & + \\
\hline $\mathrm{CoCl}_{2}$ & & - & - & - & - & - & - \\
\hline $\mathrm{CuCl}_{2}$ & & - & - & - & - & - & - \\
\hline $\mathrm{NaMoO}_{4}$ & & + & + & - & - & - & - \\
\hline $\mathrm{AlCl}_{2}$ & & + & - & - & - & - & - \\
\hline $\mathrm{CdCl}_{2}$ & & - & - & - & - & - & - \\
\hline \multirow[t]{2}{*}{$\mathrm{C}_{8} \mathrm{H}_{4} \mathrm{~K}_{2} \mathrm{O}_{12} \mathrm{Sb}_{2}$} & & - & - & - & - & - & - \\
\hline & 5B5 & & & & & & \\
\hline $\mathrm{ZnCl}_{2}$ & & + & - & - & - & - & - \\
\hline $\mathrm{NiCl}_{2}$ & & + & + & + & - & - & - \\
\hline $\mathrm{MnCl}_{2}$ & & + & + & + & + & + & + \\
\hline $\mathrm{CoCl}_{2}$ & & - & - & - & - & - & - \\
\hline $\mathrm{CuCl}_{2}$ & & - & - & - & - & - & - \\
\hline $\mathrm{NaMoO}_{4}$ & & + & + & - & - & - & - \\
\hline $\mathrm{AlCl}_{2}$ & & - & - & - & - & - & - \\
\hline $\mathrm{CdCl}_{2}$ & & - & - & - & - & - & - \\
\hline $\mathrm{C}_{8} \mathrm{H}_{4} \mathrm{~K}_{2} \mathrm{O}_{12} \mathrm{Sb}_{2}$ & & - & - & - & - & - & - \\
\hline
\end{tabular}

+, positive colony formation; -, no growth. Comparisons made with the control without metal.

ABC transporter ATP-binding protein TroB (TC 3.A.1.15.8) (Supplementary Table S5).

\section{Granulicella sp. WH15}

Cluster of Ortholog Groups analysis showed that upregulated proteins were distributed within several COG categories. The most common categories were: "G-carbohydrate transport and metabolism" (2), "M-cell wall/envelope/membrane biogenesis" (2), and "T-signal transduction mechanisms" (2) (Figure 4A). Within KEGG metabolic pathways, no complete pathway upregulation was observed.

A total of 9 upregulated proteins were assigned to KEGG orthologs, which were mapped to 12 KEGG metabolic pathways, since some orthologs were mapped to more than one pathway.
Most of the annotated proteins were mapped to "general" metabolic pathways (9), biosynthesis of antibiotics (3), and oxidative phosphorylation (3) (Figure 4B).

Some of the upregulated proteins were ATP synthase subunit $b$, and the carbohydrate-associated enzymes putative sugar phosphate isomerase YwlF (EC 5.3.1.-), UDP-glucose 4-epimerase GalE (EC 5.1.3.2) and dTP-4-dehydrorhamnose 3,5 epimerase RmlC (EC 5.1.3.13) (Supplementary Table S4). The search against BacMet databases showed that three ORFs had hits against genes related to metal ion homeostasis. ORF GWH15_13825 (cysO) had 42\% identity with predicted resistance gene $\operatorname{cop} A$, encoding a copper-exporting P-type ATPase (TC 3.A.3.5.1) and $31 \%$ similarity with the experimentally confirmed cation/multidrug efflux pump AdeG (TC 2.A.6.2.44), which is part of AdeFGH efflux system. ORF GWH15_17845 (hypothetical protein) had $30 \%$ similarity with predicted resistance gene $r c n B / y o h N$ (TC 2.A.113.1.1), a nickel/cobalt homeostasis protein; ORF GWH15_19690 (hypothetical protein) has $36 \%$ identity with predicted resistance gene $m \operatorname{tr} A$, a DNA-binding response regulator.

\section{Downregulated Proteins in Response to Manganese Granulicella sp. 5B5}

Annotation with COG database demonstrated that most proteins were distributed within the categories E-amino acid transport and metabolism (6), G-carbohydrate transport and metabolism (5), H-coenzyme transport and metabolism (4), and J-translation, ribosomal structure and biogenesis (4) (Figure 3A). Overall, 31 proteins were assigned to KEGG identifiers, which were mapped to 29 KEGG metabolic pathways. Most of the proteins were mapped to "general" metabolic pathways (29), biosynthesis of secondary metabolites (15), and biosynthesis of antibiotics (9) (Figure 3B). Several proteins linked to general metabolism were repressed, but no specific metabolic pathway seemed to be repressed. Among the repressed proteins we observed enzymes involved in various cellular functions, such as cysteine synthase CysM (EC 2.5.1.47), L-threonine dehydratase TdcB (EC 4.3.1.19), ribonucleoside-diphosphate reductase subunit beta NrdB (EC 1.17.4.1), putative glucose-6-phosphate 1-epimerase YeaD (EC 5.1.3.15) and carbonic anhydrase CynT (EC 4.2.1.1) (Supplementary Table S4).

\section{Granulicella sp. WH15}

Within COG categories, most of the downregulated proteins belonged to the categories "E-aminoacid transport and metabolism" (14), "C-energy production and conversion" (11), "J-Translation, ribosomal structure and biogenesis" (10), and "R-General function prediction" (10) (Figure 4A). Overall, 80 proteins were assigned to KEGG orthologs, which were mapped to 52 KEGG metabolic pathways, and some orthologs were mapped to more than one type of pathway. The majority of the proteins were mapped to "general" metabolic pathways (51), biosynthesis of antibiotics (21), biosynthesis of secondary metabolites (17), fatty acid metabolism (10), and microbial metabolism in diverse environments (10) (Figure 4B), with no pathway specifically stimulated. Several enzymes involved 


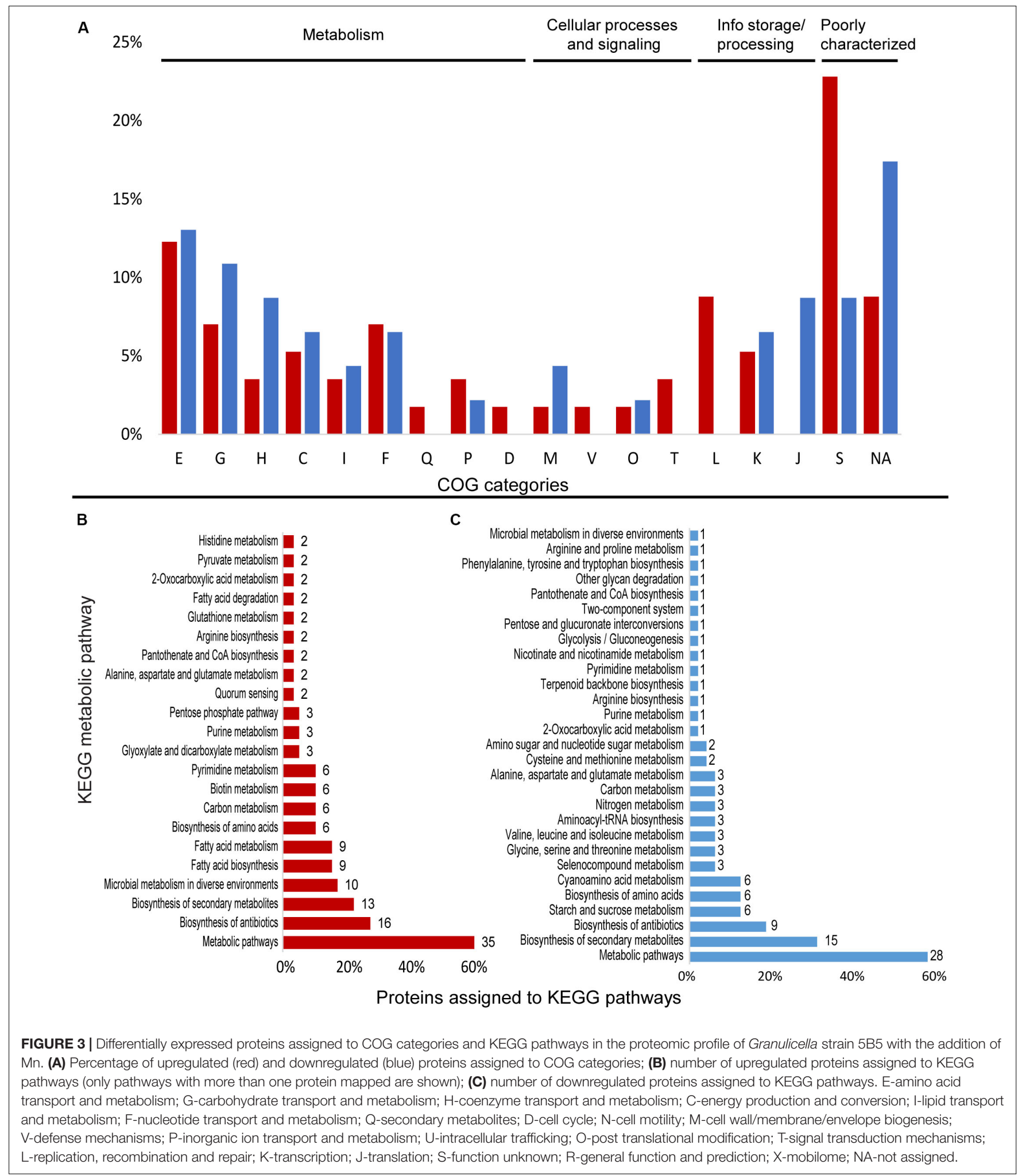

in amino acid biosynthesis and metabolism were identified, such as tyrosyl, leucyl, alanyl, and glycyl-tRNA synthetases (EC 6.1.1.-) (TyrS, LeuS, AlaS, and GlyS), leucyl aminopeptidase PepA (EC 3.4.11.1), threonine synthase ThrC (EC 4.2.3.1), xaa-pro-dipeptidyl-aminopeptidase PepQ (EC 3.4.13.9), aminopeptidase YpdF (EC 3.4.11.-), cysteine desulfurase IscS (EC 2.8.1.7), and prolyl tripeptidyl peptidase PtpA (EC 3.1.3.48) (Supplementary Table S4). 


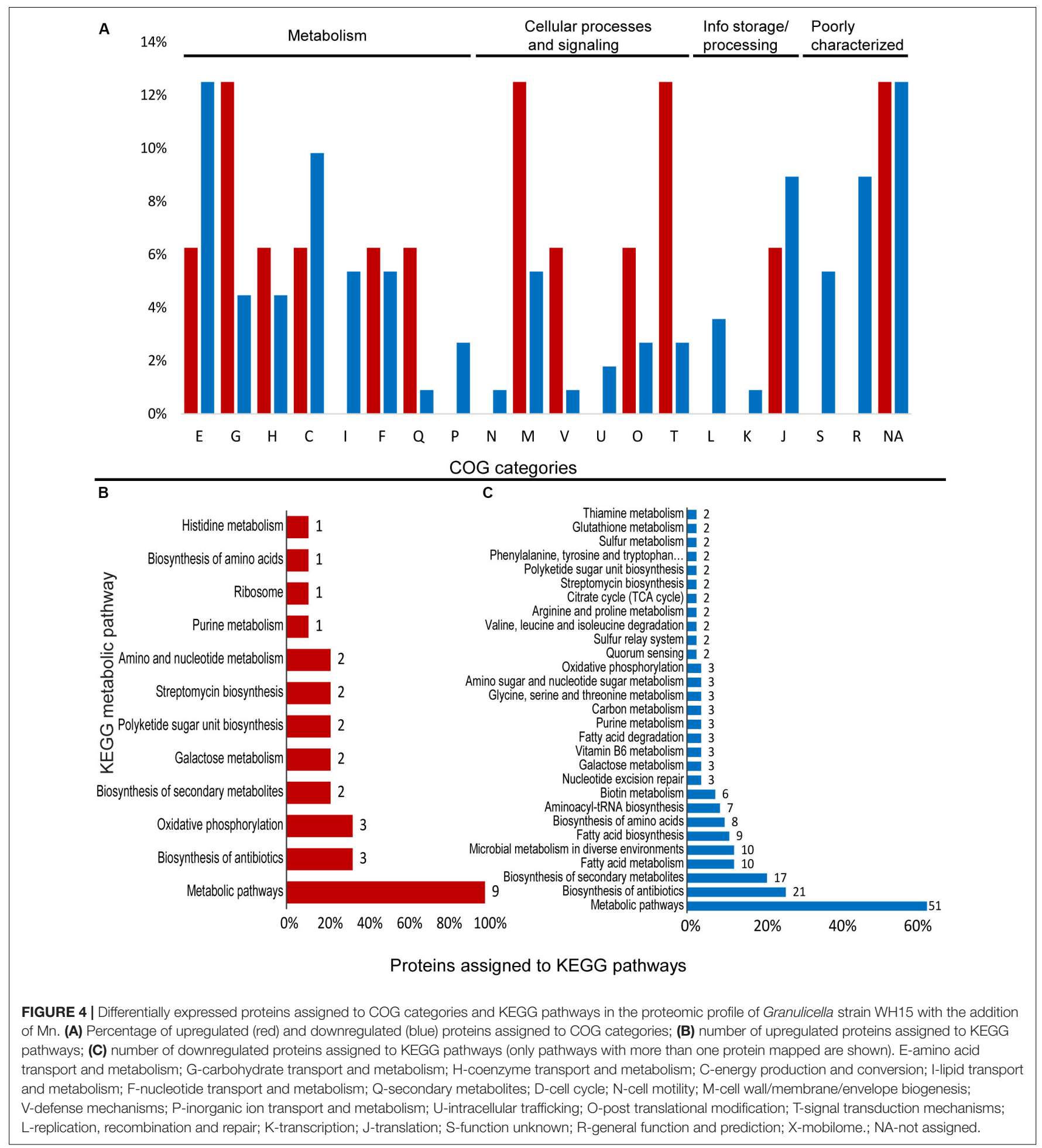

\section{DISCUSSION}

In this study, we evaluated the effect of trace element addition on the growth of two strains of Granulicella, belonging to phylum Acidobacteria class "Acidobacteriia." We observed that the growth in liquid medium of both strains was improved by the addition of Mn, to which the strains tolerated relatively higher concentrations in comparison to other metal salts. Furthermore, variations in tolerance to metal salt concentrations suggest that the Granulicella spp. 5B5 and WH15 possess different mechanisms to deal with metal ion homeostasis and stress, also reflecting the low similarity observed in the genome alignment 


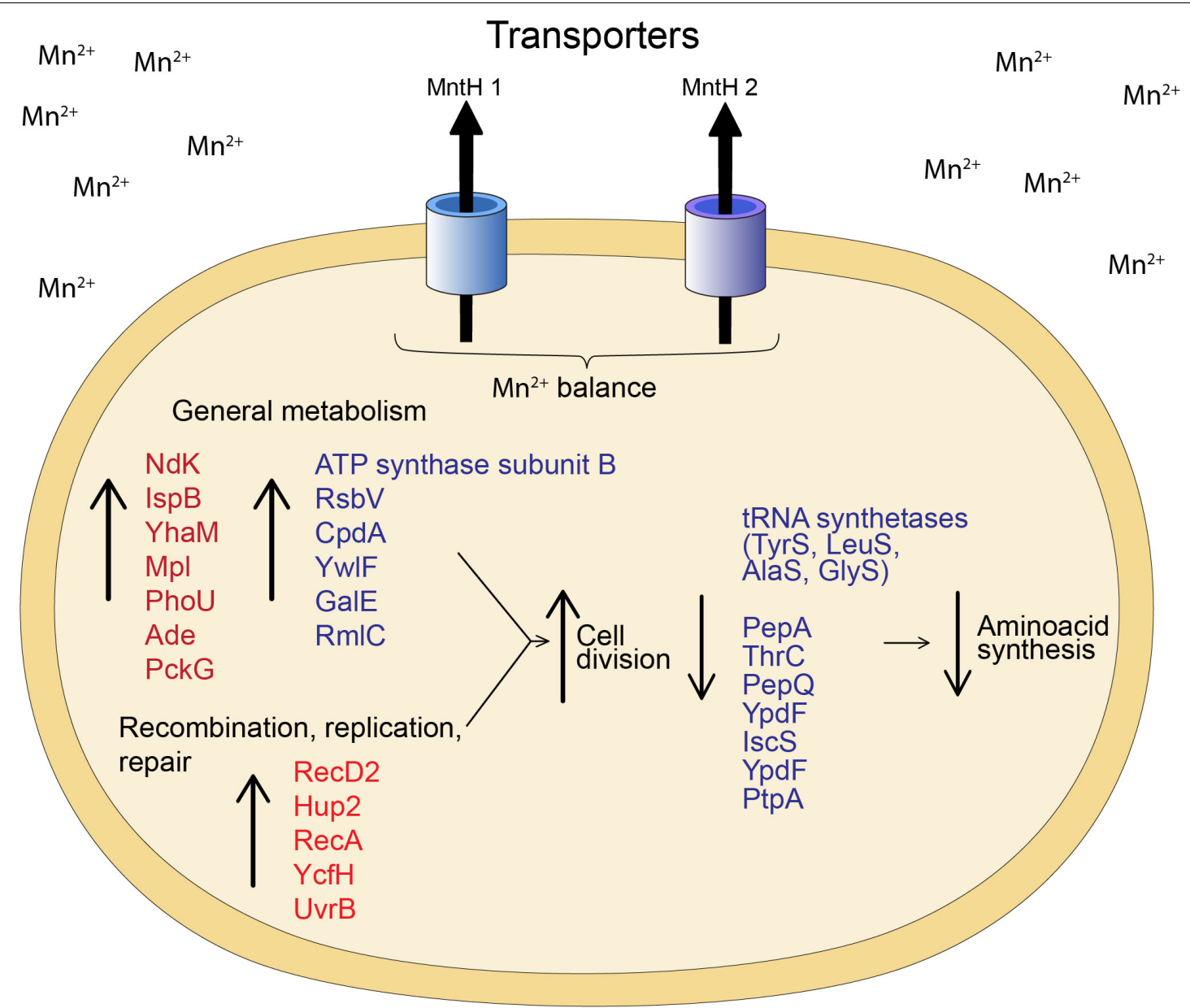

FIGURE 5 | Model of the cellular processes involved in Mn adaptation in Granulicella sp. WH15 and Granulicella sp. 5B5. Proteins in red were upregulated in Granulicella sp. 5B5, proteins in blue were up or downregulated in Granulicella sp. 5B5. The arrows depict upregulation ( $\uparrow$ ) and downregulation ( $\downarrow$ ) of proteins.

between both strains. Granulicella sp. 5B5 is likely more adapted to survive in an environment with higher concentration of several metal ions when compared to Granulicella sp. WH15.

When evaluated separately, Mn salt had a more pronounced effect than other metal salts, but the mix of metals was more effective in enhancing bacterial growth, reflecting wide physiological needs and the importance of different metal ions in bacterial metabolism. For instance, E. coli BW25113 growth was maximized with a mixture of $\mathrm{Ni}$ and $\mathrm{Fe}$, which had a better effect than each metal separately and other metal salt mixtures (Trchounian et al., 2016). The amendment of Mn to fermentation medium improved the growth of Lactobacillus bifermentans, increasing the production and activity of the enzyme glucose isomerase, necessary for biotechnological applications (Givry and Duchiron, 2008). Manganese is also an essential growth factor for Lactobacillus casei and other species of lactobacilli, which is attributed to its role as a co-factor of enzyme lactate dehydrogenase, enhancing cell growth rate and biomass concentration (Fitzpatrick et al., 2001; Lew et al., 2013). On the other hand, Mn had no significant impact in the growth of Halobacterium (Joshi et al., 2015).
Among the metals used for metal salt tolerance testing, Granulicella spp. WH15 and 5B5 only showed tolerance to Mn, exhibiting growth at the concentration of $40 \mathrm{mM} \mathrm{Mn}$, which is higher than typically observed for most bacterial strains. For instance, a resistant Serratia marcescens strain, isolated from Mn mine waters in Brazil, could grow on a maximum concentration of 6 mM Mn (Barboza et al., 2017). Mn tolerance can vary widely in microorganisms, with a minimal inhibitory concentration ranging from 0.1 to $228.9 \mathrm{mM} \mathrm{Mn}$ in certain marine bacterial strains (Gillard et al., 2019). Comparisons with other strains, however, should be interpreted cautiously, since culture media composition differ among studies, and it was already observed that organic compounds in nutrient agar can chelate metals, decreasing their availability for the microorganisms tested (Polti et al., 2007; El Baz et al., 2015).

Manganese is essential for the growth and survival of living organisms. It is a co-factor of a wide range of enzymes, being vital in specific metabolic pathways, such as sugar, lipid, and protein catabolism (Jensen and Jensen, 2014), oxygenic photosynthesis in cyanobacteria (Kehres and Maguire, 2003; Cvetkovic et al., 2010), signal transduction, stringent response, 
sporulation, and pathogenesis (Kehres and Maguire, 2003; Jensen and Jensen, 2014). One of the most widely known and studied $\mathrm{Mn}$ functions is the detoxification of ROS, where it is a redox-active co-factor in free radical detoxifying enzymes, such as Mn-superoxide dismutase (MnSod) and mangani-catalase (Jakubovics and Jenkinson, 2001; Jensen and Jensen, 2014). Additionally, the detoxifying capaticities of $\mathrm{Mn}$ are not only enzyme-mediated, since non-protein complexes of Mn can also work as antioxidants when enzymes are not sufficient (Jensen and Jensen, 2014). Nonetheless, Mn homeostasis needs a refined balance, since Mn can bind to Mg-binding sites in enzymes and regulators, causing mismetallation, which can have deleterious effects in the cells (Hohle and O'Brian, 2014; Chandrangsu et al., 2017). Both Granulicella strains were isolated from decaying wood material, in association with the white rot fungus H. fasciculare (Valášková et al., 2009), where topsoil-litter samples have $\mathrm{Mn}$ concentrations as high as $101 \mathrm{mg} \mathrm{Mn} / \mathrm{kg}$ (Costa et al., 2020a). Since high concentrations of Mn can be observed in wood decay environments, especially when decomposition is caused by white rot fungi (Blanchette, 1984), tolerance to higher manganese concentrations could be a strategy to assure the survival of the studied Acidobacteria in this environment.

The evaluation of the predicted genes in both did not reveal the presence of common genes involved in Mn regulation, such as the transcriptional regulator mntR (Jensen and Jensen, 2014). This result implies that the homeostasis of $\mathrm{Mn}$ in Granulicella strains is under control of another transcriptional regulator. MntR is a Mn-dependent DtxR family member that controls the expression of $\mathrm{Mn}$ uptake systems $m n t A B C D$ and $m n t H$ in Bacillus subtilis and Salmonella enterica (Que and Helmann, 2002; Kehres and Maguire, 2003), for instance. However, it appears that MntR is absent in Rhizobiales and Rhodobacterales, in which the function is replaced by the protein Mur (van Bakel and Wijmenga, 2005). In addition, MntR-independent regulation of $\mathrm{Mn}$ homeostasis has been proposed (Modi et al., 2011; Kaur et al., 2014). Nonetheless, both strains possessed two non-identical copies of the Mn transporter $m n t H$. MntH seems to be the main responsible transporter in Mn influx, but it was already observed that $\mathrm{Mn}$ has a significant repressive effect in the expression of Mn transporters under manganese sufficiency, keeping Mn homeostasis and optimal levels of Mn inside the cells (Guedon et al., 2003; Jensen and Jensen, 2014). Furthermore, the search of the genomes against BacMet databases demonstrated that both genomes possessed a wide range of transporters that are linked to the homeostasis of diverse metal ions.

Within the upregulated proteins from Granulicella sp. 5B5, three proteins were similar to copper P-type ATPase transporters and one protein was similar to the metal ion ATP-binding ABC transporter TroB. P-type ATPases, such as CtpC, in Mycobacterium species are responsible not only for Mn efflux, removing excess metal ion from the cells, but are also required for the metalation of proteins (Padilla-Benavides et al., 2013). ABC transporters are as well responsible for Mn uptake, important in several bacterial species (Papp-Wallace and Maguire, 2006; Jensen and Jensen, 2014). In the proteome profile of Granulicella sp. WH15, we similarly observed a protein similar to the copper-exporting P-type ATPase and cation/multidrug efflux pump AdeG, which could be involved in maintaining optimal levels of $\mathrm{Mn}$ inside the bacterial cell. Furthermore, we found a protein similar to $\mathrm{RcnB} / Y o h N$, which is an essential protein for $\mathrm{Ni} / \mathrm{Co}$ homeostasis in E. coli (Bleriot et al., 2011), and a protein similar to gene $M t r A$, which is involved in cell division control and cell wall metabolism in Mycobacterium tuberculosis (Gorla et al., 2018).

In addition to transporters, the proteomic analyses of both bacteria strains revealed other proteins which might be involved in the growth enhancement of both strains. The proteomic profile of Granulicella sp. WH15 had fewer upregulated enzymes in comparison to Granulicella sp. 5B5, demonstrating that $\mathrm{Mn}$ had more impact in transcription and protein expression in Granulicella sp. 5B5. Several enzymes that require $\mathrm{Mn}$ or $\mathrm{Mg}$ as cofactor were upregulated in the proteome of Granulicella sp. 5B5, but no enhancement of specific metabolic pathway was detected. Overall, the upregulated enzymes in Granulicella sp. 5B5 were involved in general metabolic pathways, which could be stimulated due to the higher metabolism necessary for a faster growth. For instance, nucleoside diphosphate kinase Ndk is a critical enzyme involved in the nucleotide metabolism of microorganisms, but is also part of posttranslational modification of proteins, as well as regulation of genes linked to quorum sensing, proteases and toxins ( $\mathrm{Yu}$ et al., 2017). Octaprenyl diphosphate synthase IspB is involved in the production of the lateral chain of ubiquinones, and is an essential enzyme for respiration and normal growth of $E$. coli (Okada et al., 1997). $3^{\prime}-5^{\prime}$ exoribonuclease YhaM was identified as a participating enzyme in mRNA turnover in B. subtilis (Oussenko et al., 2005). UDP-N-acetylmuramate-L-alanylgamma-D-glutamyl-meso-2,6-diaminoheptandioate ligase $\mathrm{Mpl}$ is a recycling enzyme that allows the constant remodeling of bacterial cell wall polymer occurring during cell growth and division (Herve et al., 2007). Moreover, the category replication, recombination and repair were only present within upregulated proteins, with proteins DNA helicase RecD2, DNA -binding protein Hup 2, protein RecA, metal-dependent hydrolase $\mathrm{YcfH}$ and UvrABC system protein UvrB. In addition, no specific pathway seemed to be repressed.

In the proteomic profile of Granulicella sp. WH15, we observed the upregulation enzymes involved in energy production, amino acid metabolism and transcription regulation. For instance, ATP synthase subunit $\beta$ is part of the ATP synthase complex, which is involved in ATP synthesis and hydrolysis (Sokolov et al., 1999). ATP phosphoribosyltransferase is an enzyme involved in histidine biosynthesis, a reaction that requires $\mathrm{Mg}$, which can be substituted by $\mathrm{Mn}$ (Tebar et al., 1977). Protein RsbV is a positive regulator of factor sigma $\beta$, which, in Gram-positive bacteria is a key contributor to the resilience and survival of bacterial species to environmental conditions, such as variations in $\mathrm{pH}$, osmotic stress or entry into stationary growth phase (Kullik and Giachino, 1997; Guldimann et al., 2016). Phosphodiesterase CpdA is responsible for the hydrolysis of the second messenger cyclic AMP (cAMP), which controls cell responses to a variety of environmental conditions (Fuchs et al., 2010). Similarly to the response of S. marcescens (Queiroz et al., 2018) 
to $\mathrm{Mn}$ stimulation, the limited number of upregulated proteins from Granulicella sp. WH15 could be attributed to the adaptation to the concentration of $\mathrm{Mn}$ used in the experiment, since it is an optimal growth condition. Furthermore, as a co-factor of several important enzymes (Crowley et al., 2000; Jensen and Jensen, 2014), the presence of manganese might be improving bacterial growth by activating enzymes and enhancing metabolic activities involved in cell cycle and division, even when the enzymes were not differentially expressed. Among the repressed proteins, we found several amino acid synthases, that could be inhibited due to the ready availability of amino acids in the culture medium composition (Mader et al., 2002), supplied by yeast extract.

The proteomic profiles of both strains did not exhibit the overexpression of specific pathways, indicating that $\mathrm{Mn}$ was more important in enhance enzymatic activity than to protein expression regulation. Finally, we did not find the most common transcriptional regulation of $\mathrm{Mn}$ homeostasis, implying that $\mathrm{Mn}$ regulation is performed by a different gene or set of genes, but our analysis revealed candidate transporters that could be potentially involved in Mn homeostasis for Granulicella species. The presence of such type of transporters could facilitate the uptake of metal ions, improving the adaptability of bacteria to metal enriched environments (Campanharo et al., 2016), promoting a tight regulation of metal ion homeostasis, as well as a tolerance to higher concentrations of metals.

\section{DATA AVAILABILITY STATEMENT}

The Granulicella sp. 5B5 strain genome is deposited at NCBI with accession number CP046444. The Granulicella sp. WH15 strain genome is deposited at NCBI with accession number CP042596. The mass spectrometry proteomics data were deposited to

\section{REFERENCES}

Abbas, A. S., and Edwards, C. (1990). Effects of metals on Streptomyces coelicolor growth and actinorhodin production. Appl. Environ. Microbiol. 56, 675-680. doi: 10.1128/aem.56.3.675-680.1990

Abou-Shanab, R. A. I., Van Berkum, P., and Angle, J. S. (2007). Heavy metal resistance and genotypic analysis of metal resistance genes in gram-positive and gram-negative bacteria present in Ni-rich serpentine soil and in the rhizosphere of Alyssum murale. Chemosphere 68, 360-367. doi: 10.1016/j.chemosphere. 2006.12.051

Afzal, A. M., Rasool, M. H., Waseem, M., and Aslam, B. (2017). Assessment of heavy metal tolerance and biosorptive potential of Klebsiella variicola isolated from industrial effluents. AMB Express 7:184.

Ankenbrand, M. J., Hohlfeld, S., Hackl, T., and Förster, F. (2017). AliTVinteractive visualization of whole genome comparisons. PeerJ Comp. Sci. 3:e116. doi: 10.7717/peerj-cs.116

Aziz, R. K., Bartels, D., Best, A. A., Dejongh, M., Disz, T., Edwards, R. A., et al. (2008). The RAST server: rapid annotations using subsystems technology. BMC Genomics 9:75. doi: 10.1186/1471-2164-9-75

Banerjee, D., Jana, M., and Mahapatra, S. (2009). Production of exopolysaccharide by endophytic Stemphylium sp. Micol. Aplicada Int. 21:6.

Barboza, N. R., Morais, M. M. C. A., Queiroz, P. S., Amorim, S. S., Guerra-Sá, R., and Leão, V. A. (2017). High manganese tolerance and biooxidation ability the ProteomeXchange Consortium (http://proteomecentral. proteomexchange.org) via the PRIDE (Perez-Riverol et al., 2019) partner repository with the dataset identifier PXD016551.

\section{AUTHOR CONTRIBUTIONS}

EK and OC: conceptualization. OC, CO, DZ, CW, and KR: methodology. OC, EK, and DZ: data analysis and integration. OC: writing - original draft preparation. EK, OC, $\mathrm{CB}$, and DZ: writing - review and editing. All authors have read and agreed to the published version of the manuscript.

\section{FUNDING}

OC was supported by SWB grant from CNPq (202496/2015-5) (Conselho Nacional de Desenvolvimento Científico e Tecnológico).

\section{ACKNOWLEDGMENTS}

We thank Victor Jager and Mattias de Hollander for bioinformatics assistance, Genomics Resource Center (United States) for the bacteria genome sequencing and Jos Raaijmakers and George Kowalchulk for comments on the manuscript. Publication number 6973 of Netherlands Institute of Ecology (NIOO-KNAW).

\section{SUPPLEMENTARY MATERIAL}

The Supplementary Material for this article can be found online at: https://www.frontiersin.org/articles/10.3389/fmicb. 2020.01227/full\#supplementary-material

of Serratia marcescens isolated from manganese mine water in Minas Gerais, Brazil. Front. Microbiol. 8:1946. doi: 10.3389/fmicb.2017.01946

Blanchette, R. A. (1984). Manganese accumulation in wood decayed by white rot fungi. Phytopathology 74, 725-730.

Bleriot, C., Effantin, G., Lagarde, F., Mandrand-Berthelot, M. A., and Rodrigue, A. (2011). RcnB Is a periplasmic protein essential for maintaining intracellular $\mathrm{Ni}$ and Co concentrations in Escherichia coli. J. Bacteriol. 193, 3785-3793. doi: 10.1128/JB.05032-11

Campanharo, J. C., Kielak, A. M., Castellane, T. C. L., Kuramae, E. E., and Lemos, E. G. D. M. (2016). Optimized medium culture for Acidobacteria subdivision 1 strains. FEMS Microbiol. Lett. 363:fnw245. doi: 10.1093/femsle/fnw245

Chandrangsu, P., Rensing, C., and Helmann, J. D. (2017). Metal homeostasis and resistance in bacteria. Nat. Rev. Microbiol. 15, 338-350. doi: 10.1038/nrmicro. 2017.15

Chin, C.-S., Alexander, D. H., Marks, P., Klammer, A. A., Drake, J., Heiner, C., et al. (2013). Nonhybrid, finished microbial genome assemblies from long-read SMRT sequencing data. Nat. Methods 10, 563-569. doi: 10.1038/nmeth.2474

Costa, O. Y. A., De Hollander, M., Pijl, A., and Kuramae, E. E. (2020a). Cultivationindependent and cultivation-dependent metagenomes reveal genetic and enzymatic potential of microbial community involved in the degradation of a complex microbial polymer. Microbiome (in press).

Costa, O. Y. A., Zerillo, M. M., Zuhlke, D., Kielak, A. M., Pijl, A., Riedel, K., (2020b). Responses of Acidobacteria granulicella Sp. WH15 to high carbon 
revealed by integrated omics analyses. Microorganisms 8:244. doi: 10.3390/ microorganisms 8020244

Crowley, J. D., Traynor, D. A., and Weatherburn, D. C. (2000). Enzymes and proteins containing manganese: an overview. Met. Ions Biol. Syst. 37, 209-278.

Cvetkovic, A., Menon, A. L., Thorgersen, M. P., Scott, J. W., Poole, F. L. II, Jenney, F. E. Jr., et al. (2010). Microbial metalloproteomes are largely uncharacterized. Nature 466, 779-782. doi: 10.1038/nature09265

de Castro, V. H. L. (2011). Identificação, Isolamento e Caracterização de Bactérias de Solo de Cerrado Pertencentes ao filo Acidobacteria. Ph.D. Dissertation, Universidade Católica de Brasília, Brasília.

de Castro, V. H. L., Schroeder, L. F., Quirino, B. F., Kruger, R. H., and Barreto, C. C. (2013). Acidobacteria from oligotrophic soil from the Cerrado can grow in a wide range of carbon source concentrations. Can. J. Microbiol. 59, 746-753. doi: 10.1139/cjm-2013-0331

Dedysh, S. N., and Yilmaz, P. (2018). Refining the taxonomic structure of the phylum Acidobacteria. Int. J. Syst. Evol. Microbiol. 68, 3796-3806. doi: 10.1099/ ijsem.0.003062

Eichorst, S. A., Kuske, C. R., and Schmidt, T. M. (2011). Influence of plant polymers on the distribution and cultivation of bacteria in the phylum Acidobacteria. Appl. Environ. Microbiol. 77, 586-596. doi: 10.1128/AEM.01080-10

El Baz, S., Baz, M., Barakate, M., Hassani, L., El Gharmali, A., and Imziln, B. (2015). Resistance to and accumulation of heavy metals by actinobacteria isolated from abandoned mining areas. Sci. World J. 2015, 1-14. doi: 10.1155/2015/ 761834

Figueras, M. J., Beaz-Hidalgo, R., Hossain, M. J., and Liles, M. R. (2014). Taxonomic affiliation of new genomes should be verified using average nucleotide identity and multilocus phylogenetic analysis. Genome Announc. 2:e00927-14.

Fitzpatrick, J. J., Ahrens, M., and Smith, S. (2001). Effect of manganese on Lactobacillus casei fermentation to produce lactic acid from whey permeate. Process Biochem. 36, 671-675. doi: 10.1016/s0032-9592(00) 00265-x

Foesel, B. U., Rohde, M., and Overmann, J. (2013). Blastocatella fastidiosa gen. nov., sp. nov., isolated from semiarid savanna soil-The first described species of Acidobacteria subdivision 4. Syst. Appl. Microbiol. 36, 82-89. doi: 10.1016/j. syapm.2012.11.002

Fuchs, E. L., Brutinel, E. D., Klem, E. R., Fehr, A. R., Yahr, T. L., and Wolfgang, M. C. (2010). In vitro and in vivo vharacterization of the Pseudomonas aeruginosa Cyclic AMP (cAMP) Phosphodiesterase CpdA, required for cAMP homeostasis and virulence factor regulation. J. Bacteriol. 192, 2779-2790. doi: 10.1128/JB. 00168-10

Galperin, M. Y., Makarova, K. S., Wolf, Y. I., and Koonin, E. V. (2015). Expanded microbial genome coverage and improved protein family annotation in the COG database. Nucleic Acids Res. 43, D261-D269. doi: 10.1093/nar/ gku1223

Gillard, B., Chatzievangelou, D., Thomsen, L., and Ullrich, M. S. (2019). Heavy-metal-resistant microorganisms in deep-sea sediments disturbed by mining activity: an application toward the development of experimental in vitro systems. Front. Mar. Sci. 6:462. doi: 10.3389/fmars.2019. 00462

Givry, S., and Duchiron, F. (2008). Optimization of culture medium and growth conditions for production of L-arabinose isomerase and D-xylose isomerase by Lactobacillus bifermentans. Microbiology 77, 281-287. doi: 10 . 1134/s0026261708030053

Gorla, P., Plocinska, R., Sarva, K., Satsangi, A. T., Pandeeti, E., Donnelly, R., et al. (2018). MtrA response regulator controls cell division and cell wall metabolism and affects susceptibility of mycobacteria to the first line antituberculosis drugs. Front. Microbiol. 9:2839. doi: 10.3389/fmicb.2018.02839

Grube, M., Cernava, T., Soh, J., Fuchs, S., Aschenbrenner, I., Lassek, C., et al. (2014). Exploring functional contexts of symbiotic sustain within lichen-associated bacteria by comparative omics. ISME J. 9, 412-424. doi: 10.1038/ismej. 2014.138

Guedon, E., Moore, C. M., Que, Q., Wang, T., Ye, R. W., and Helmann, J. D. (2003). The global transcriptional response of Bacillus subtilis to manganese involves the MntR, Fur, TnrA and $\sigma \mathrm{B}$ regulons. Mol. Microbiol. 49, 1477-1491. doi: 10.1046/j.1365-2958.2003.03648.x

Guldimann, C., Boor, K. J., Wiedmann, M., Guariglia-Oropeza, V., and Schaffner, D. W. (2016). Resilience in the face of uncertainty: sgma factor B fine-tunes gene expression to support homeostasis in gram-positive bacteria. Appl. Environ. Microbiol. 82, 4456-4469. doi: 10.1128/AEM.00714-16

Hantke, K. (2001). Iron and metal regulation in bacteria. Curr. Opin. Microbiol. 4:5.

Harris, R. S. (2007). Improved Pairwise Alignment of Genomic DNA. Ph.D. Thesis, Pennsylvania State University, State College, PA.

Herve, M., Boniface, A., Gobec, S., Blanot, D., and Mengin-Lecreulx, D. (2007). Biochemical characterization and physiological properties of Escherichia coli UDP-N-Acetylmuramate:L-Alanyl- -D-Glutamyl-meso- diaminopimelate ligase. J. Bacterio. 189, 3987-3995. doi: 10.1128/jb.00087-07

Hohle, T. H., and O'Brian, M. R. (2014). Magnesium-dependent processes are targets of bacterial manganese toxicity. Mol. Microbiol. 93, 736-747. doi: 10. $1111 / \mathrm{mmi} .12687$

Huerta-Cepas, J., Forslund, K., Coelho, L. P., Szklarczyk, D., Jensen, L. J., Von Mering, C., et al. (2017). Fast genome-wide functional annotation through orthology assignment by eggNOG-mapper. Mol. Biol. Evol. 34, 2115-2122. doi: 10.1093/molbev/msx148

Jakubovics, N. S., and Jenkinson, H. F. (2001). Out of the iron age: new insights into the critical role of manganese homeostasis in bacteria. Microbiology 147, 1709-1718. doi: 10.1099/00221287-147-7-1709

Janssen, P. H., Yates, P. S., Grinton, B. E., Taylor, P. M., and Sait, M. (2002). Improved culturability of soil bacteria and isolation in pure culture of novel members of the divisions Acidobacteria, Actinobacteria, Proteobacteria, and Verrucomicrobia. Appl. Environ. Microbiol. 68, 2391-2396. doi: 10.1128/aem. 68.5.2391-2396.2002

Jensen, A. N., and Jensen, L. T. (2014). "CHAPTER 1. Manganese transport, trafficking and function in invertebrates," in Manganese in Health and Disease, eds G. C. Lucio and A. Michael (Cambridge: Royal Society of Chemistry), 1-33. doi: 10.1039/9781782622383-00001

Joshi, P. A., Singh, N., and Shekhawat, D. B. (2015). Effect of metal ions on growth and biosurfactant production by halophilic bacteria. Adv. Appl. Sci. Res. 6:4.

Kanehisa, M. (2000). KEGG: kyoto encyclopedia of genes and genomes. Nucleic Acids Res. 28, 27-30.

Kaur, G., Sengupta, S., Kumar, V., Kumari, A., Ghosh, A., Parrack, P., et al. (2014). Novel MntR-independent mechanism of manganese homeostasis in Escherichia coli by the ribosome-associated protein HflX. J. Bacteriol. 196, 2587-2597. doi: 10.1128/JB.01717-14

Kehres, D. G., and Maguire, M. E. (2003). Emerging themes in manganese transport, biochemistry and pathogenesis in bacteria. FEMS Microbiol. Rev. 27, 263-290. doi: 10.1016/s0168-6445(03)00052-4

Kullik, I., and Giachino, P. (1997). The alternative sigma factor $\sigma$ B in Staphylococcus aureus : regulation of the sigB operon in response to growth phase and heat shock. Arch. Microbiol. 167, 151-159. doi: 10.1007/ s002030050428

Kumar, S., Stecher, G., Li, M., Knyaz, C., Tamura, K., and Battistuzzi, F. U. (2018). MEGA X: molecular evolutionary genetics analysis across computing platforms. Mol. Biol. Evol. 35, 1547-1549. doi: 10.1093/molbev/ msy096

Kuramae, E. E., Yergeau, E., Wong, L. C., Pijl, A. S., Veen, J. A., and Kowalchuk, G. A. (2012). Soil characteristics more strongly influence soil bacterial communities than land-use type. FEMS Microbiol. Ecol. 79, 12-24. doi: 10.1111/ j.1574-6941.2011.01192.x

Lew, L. C., Liong, M. T., and Gan, C. Y. (2013). Growth optimization of Lactobacillus rhamnosus FTDC 8313 and the production of putative dermal bioactives in the presence of manganese and magnesium ions. J. Appl. Microbiol. 114, 526-535. doi: 10.1111/jam.12044

Locatelli, F. M., Goo, K. S., and Ulanova, D. (2016). Effects of trace metal ions on secondary metabolism and the morphological development of streptomycetes. Metallomics 8, 469-480. doi: 10.1039/c5mt00324e

Lombard, V., Golaconda Ramulu, H., Drula, E., Coutinho, P. M., and Henrissat, B. (2014). The carbohydrate-active enzymes database (CAZy) in 2013. Nucleic Acids Res. 42, D490-D495. doi: 10.1093/nar/gkt1178

Mader, U., Homuth, G., Scharf, C., Buttner, K., Bode, R., and Hecker, M. (2002). Transcriptome and proteome analysis of Bacillus subtilis gene expression modulated by amino acid availability. J. Bacteriol. 184, 4288-4295. doi: 10.1128/ jb.184.15.4288-4295.2002 
Merchant, S. S., and Helmann, J. D. (2012). Elemental economy: microbial strategies for optimizing growth in the face of nutrient limitation. Adv. Microb. Physiol. 60, 91-210. doi: 10.1016/b978-0-12-398264-3.00002-4

Modi, S. R., Camacho, D. M., Kohanski, M. A., Walker, G. C., and Collins, J. J. (2011). Functional characterization of bacterial sRNAs using a network biology approach. Proc. Natl. Acad. Sci. U.S.A. 108, 15522-15527. doi: 10.1073/pnas. 1104318108

Morgenstern, A., Paetz, C., Behrend, A., and Spiteller, D. (2015). Divalent transition-metal-ion stress induces prodigiosin biosynthesis in Streptomyces coelicolor m145: formation of coeligiosins. Chem. A Eur. J. 21, 6027-6032. doi: 10.1002/chem.201405733

Navarrete, A. A., Barreto, C. C., Arnaldo, M., and Tsai, S. M. (2013a). Molecular detection on culture medium of Acidobacteria from Amazon soils. Microbiol. Discov. 1, 607-621.

Navarrete, A. A., Kuramae, E. E., De Hollander, M., Pijl, A. S., Van Veen, J. A., and Tsai, S. M. (2013b). Acidobacterial community responses to agricultural management of soybean in Amazon forest soils. FEMS Microbiol. Ecol. 83, 607-621. doi: 10.1111/1574-6941.12018

NCBI Resource Coordinators (2016). Database resources of the national center for biotechnology information. Nucleic Acids Res. 44, D7-D19. doi: 10.1093/nar/ gkv1290

Okada, K., Minehira, M., Zhu, X., Suzuki, K., Nakagawa, T., Matsuda, H., et al. (1997). The ispB gene encoding octaprenyl diphosphate synthase is essential for growth of Escherichia coli. J. Bacteriol. 179, 3058-3060. doi: 10.1128/jb.179.9. 3058-3060.1997

Oussenko, I. A., Abe, T., Ujiie, H., Muto, A., and Bechhofer, D. H. (2005). Participation of 3'-to-5' exoribonucleases in the turnover of Bacillus subtilis mRNA. J. Bacteriol. 187, 2758-2767. doi: 10.1128/jb.187.8.2758-2767.2005

Padilla-Benavides, T., Long, J. E., Raimunda, D., Sassetti, C. M., and Argüello, J. M. (2013). A Novel P1B-type Mn2+-transporting ATPase is required for secreted protein metallation in mycobacteria. J. Biol. Chem. 288, 11334-11347. doi: $10.1074 /$ jbc.m112.448175

Pal, C., Bengtsson-Palme, J., Rensing, C., Kristiansson, E., and Larsson, D. G. J. (2014). BacMet: antibacterial biocide and metal resistance genes database. Nucleic Acids Res. 42, D737-D743. doi: 10.1093/nar/gkt1252

Pankratov, T. A., Serkebaeva, Y. M., Kulichevskaya, I. S., Liesack, W., and Dedysh, S. N. (2008). Substrate-induced growth and isolation of Acidobacteria from acidic Sphagnum peat. ISME J. 2, 551-560. doi: 10.1038/ismej. 2008.7

Papp-Wallace, K. M., and Maguire, M. E. (2006). Manganese transport and the role of manganese in virulence. Annu. Rev. Microbiol. 60, 187-209. doi: 10.1146/ annurev.micro.60.080805.142149

Pereira de Castro, A., Sartori, Da Silva, M. R. S., Quirino, B. F., Da Cunha, Bustamante, M. M., et al. (2016). Microbial diversity in cerrado biome (neotropical savanna) soils. PLoS One 11:e0148785. doi: 10.1371/journal.pone. 0148785

Perez-Riverol, Y., Csordas, A., Bai, J., Bernal-Llinares, M., Hewapathirana, S., Kundu, D. J., et al. (2019). The PRIDE database and related tools and resources in 2019: improving support for quantification data. Nucleic Acids Res. 47, D442-D450.

Polti, M. A., Amoroso, M. J., and Abate, C. M. (2007). Chromium(VI) resistance and removal by actinomycete strains isolated from sediments. Chemosphere 67, 660-667. doi: 10.1016/j.chemosphere.2006.11.008

Puri, S., Hohle, T. H., and O'brian, M. R. (2010). Control of bacterial iron homeostasis by manganese. Proc. Natl. Acad. Sci. U.S.A. 107, 10691-10695. doi: $10.1073 /$ pnas. 1002342107

Que, Q., and Helmann, J. D. (2002). Manganese homeostasis in Bacillus subtilis is regulated by $\mathrm{MntR}$, a bifunctional regulator related to the diphtheria toxin repressor family of proteins. Mol. Microbiol. 35, 1454-1468. doi: 10.1046/j. 1365-2958.2000.01811.x

Queiroz, P. S., Ruas, F. A. D., Barboza, N. R., De Castro Borges, W., and Guerra-Sá, R. (2018). Alterations in the proteomic composition of Serratia marcescens in response to manganese (II). BMC Biotechnol. 18:83. doi: 10.1186/s12896-0180493-3

Rawat, S. R., Männistö, M. K., Starovoytov, V., Goodwin, L., Nolan, M., Hauser, L., et al. (2013a). Complete genome sequence of Granulicella tundricola type strain MP5ACTX9T, an Acidobacteria from tundra soil. Stand. Genomic Sci. 9, 449-461. doi: 10.4056/sigs.4648353
Rawat, S. R., Männistö, M. K., Starovoytov, V., Goodwin, L., Nolan, M., Hauser, L. J., et al. (2013b). Complete genome sequence of Granulicella mallensis type strain MP5ACTX8T, an acidobacterium from tundra soil. Stand. Genomic Sci. 9, 71-82. doi: 10.4056/sigs.4328031

Saeed, A. I., Sharov, V., White, J., Li, J., Liang, W., Bhagabati, N., et al. (2003). TM4: a free,open-source system for microarray data management and analysis. BioTechniques 34, 374-378. doi: 10.2144/03342mt01

Seemann, T. (2014). Prokka: rapid prokaryotic genome annotation. Bioinformatics 30, 2068-2069. doi: 10.1093/bioinformatics/ btu153

Sokolov, M., Lu, L., Tucker, W., Gao, F., Gegenheimer, P. A., and Richter, M. L. (1999). The 20 C-terminal amino acid residues of the chloroplast ATP synthase $\gamma$ subunit are not essential for activity. J. Biol. Chem. 274, 13824-13829. doi: $10.1074 /$ jbc. 274.20 .13824

Stevenson, B. S., Eichorst, S. A., Wertz, J. T., Schmidt, T. M., and Breznak, J. A. (2004). New strategies for cultivation and detection of previously uncultured microbes. Appl. Environ. Microbiol. 70, 4748-4755. doi: 10.1128/aem.70.8. 4748-4755.2004

Stopnisek, N., Zühlke, D., Carlier, A., Barberán, A., Fierer, N., Becher, D., et al. (2016). Molecular mechanisms underlying the close association between soil Burkholderia and fungi. ISME J. 10, 253-264. doi: 10.1038/ismej.2 015.73

Stothard, P., and Wishart, D. S. (2004). Circular genome visualization and exploration using CGView. Bioinformatics 21, 537-539. doi: 10.1093/ bioinformatics/bti054

Tamura, K., and Nei, M. (1993). Estimation of the number of nucleotide substitutions in the control region of mitochondrial-DNA in humans and chimpanzees. Mol. Biol. Evol. 10, 512-526.

Tebar, A. R., Ballesteros, A., and Soria, J. (1977). Mn2+ electron spin resonance studies on ATP phosphoribosyltransferase from E. coli. Experientia 33, 1292-1294. doi: 10.1007/bf01920140

Terry, L. R., Kulp, T. R., Wiatrowski, H., Miller, L. G., Oremland, R. S., and Drake, H. L. (2015). Microbiological oxidation of antimony(III) with oxygen or nitrate by bacteria isolated from contaminated mine sediments. Appl. Environ. Microbiol. 81, 8478-8488. doi: 10.1128/AEM.019 $70-15$

Trchounian, K., Poladyan, A., and Trchounian, A. (2016). Optimizing strategy for Escherichia coli growth and hydrogen production during glycerol fermentation in batch culture: effects of some heavy metal ions and their mixtures. Appl. Energy 177, 335-340. doi: 10.1016/j.apenergy.2016. 05.129

Valášková, V., De Boer, W., Klein Gunnewiek, P. J. A., Pospíšek, M., and Baldrian, P. (2009). Phylogenetic composition and properties of bacteria coexisting with the fungus Hypholoma fasciculare in decaying wood. ISME J. 3, 1218-1221. doi: 10.1038/ismej.2009.64

van Bakel, H., and Wijmenga, C. (2005). "Family matters: gene regulation by metal-dependent transcription factors," in Molecular Biology of Metal Homeostasis and Detoxification. Topics in Current Genetics, Vol. 14, eds M. J. Tamas and E. Martinoia (Berlin: Springer), 341-394. doi: 10.1007/ 4735_104

Walker, B. J., Abeel, T., Shea, T., Priest, M., Abouelliel, A., Sakthikumar, S., et al. (2014). Pilon: an integrated tool for comprehensive microbial variant detection and genome assembly improvement. PLoS One 9:e112963. doi: 10.1371/journal. pone.0112963

Widdel, F., Kohring, G.-W., and Mayer, F. (1983). Studies on dissimilatory sulfatereducing bacteria that decompose fatty acids. Arch. Microbiol. 134, 286-294. doi: $10.1007 /$ bf00407804

Wintsche, B., Glaser, K., Sträuber, H., Centler, F., Liebetrau, J., Harms, H., et al. (2016). Trace elements induce predominance among methanogenic activity in anaerobic digestion. Front. Microbiol. 7:2034. doi: 10.3389/fmicb.2016. 02034

Yoon, S.-H., Ha, S.-M., Lim, J., Kwon, S., and Chun, J. (2017). A large-scale evaluation of algorithms to calculate average nucleotide identity. Antonie Van Leeuwenhoek 110, 1281-1286. doi: 10.1007/s10482-017-0844-4

Yu, H., Rao, X., and Zhang, K. (2017). Nucleoside diphosphate kinase (Ndk): a pleiotropic effector manipulating bacterial virulence and adaptive responses. Microbiol. Res. 205, 125-134. doi: 10.1016/j.micres.2017. 09.001 
Zhang, H., Yohe, T., Huang, L., Entwistle, S., Wu, P., Yang, Z., et al. (2018). dbCAN2: a meta server for automated carbohydrate-active enzyme annotation. Nucleic Acids Res. 46, W95-W101. doi: 10.1093/nar/ gky418

Zhang, Y., Rodionov, D. A., Gelfand, M. S., and Gladyshev, V. N. (2009). Comparative genomic analyses of nickel, cobalt and vitamin B12 utilization. BMC Genomics 10:78. doi: 10.1186/1471-2164-10-78

Zhang, Y., Wen, Z., Washburn, M. P., and Florens, L. (2010). Refinements to label free proteome quantitation: how to deal with peptides shared by multiple proteins. Anal. Chem. 82, 2272-2281. doi: 10.1021/ac902 3999
Conflict of Interest: The authors declare that the research was conducted in the absence of any commercial or financial relationships that could be construed as a potential conflict of interest.

Copyright (c) 2020 Costa, Oguejiofor, Zühlke, Barreto, Wünsche, Riedel and Kuramae. This is an open-access article distributed under the terms of the Creative Commons Attribution License (CC BY). The use, distribution or reproduction in other forums is permitted, provided the original author(s) and the copyright owner(s) are credited and that the original publication in this journal is cited, in accordance with accepted academic practice. No use, distribution or reproduction is permitted which does not comply with these terms. 